\title{
On the asymptotic optimality of error bounds for some linear complementarity problems
}

\author{
M. García-Esnaola · J. M. Peña
}

Received: date / Accepted: date

\begin{abstract}
We introduce strong $B$-matrices and strong $B$-Nekrasov matrices, for which some error bounds for linear complementarity problems are analyzed. In particular, it is proved that the bounds of [5] and of [8] are asymptotically optimal for strong $B$-matrices and strong $B$-Nekrasov matrices, respectively. Other comparisons with a bound of [11] are performed.

Keywords Error bounds $\cdot$ Linear complementarity problems $\cdot B$-matrices $\cdot B$-Nekrasov matrices $\cdot P$-matrices
\end{abstract}

Mathematics Subject Classification (2000) 90C33 - 90C31 - 65G50 $\cdot 15 \mathrm{~A} 48$

\section{Introduction}

The linear complementarity problem $(\operatorname{LCP}(M, q))$ looks for a vector $x \in \mathbf{R}^{n}$ such that

$$
M x+q \geq 0, \quad x \geq 0, \quad x^{T}(M x+q)=0,
$$

where $M$ is an $n \times n$ real matrix and $q \in \mathbf{R}^{n}$.

It is well known that this problem has a unique solution if and only if $M$ has positive principal minors (i.e., $M$ is a $P$-matrix). Important applications of this problem can be seen in [2].

Research partially supported by the Spanish Research Grant MTM2015-65433-P (MINECO/FEDER) and by Gobierno de Aragón and Fondo Social Europeo.

M. García-Esnaola

Departamento de Matemática Aplicada

Universidad de Zaragoza

Tel.: (+34) 976761985

E-mail: gesnaola@unizar.es

Juan Manuel Peña

Departamento de Matemática Aplicada

Universidad de Zaragoza

Tel.: (+34) 976761129

E-mail: jmpena@unizar.es 
Error bounds for LCP of $P$-matrices have been studied (cf., [1], [12]). For particular subclasses of $P$-matrices, the bounds can be refined: see [1] and [6] for the subclass of $\mathrm{H}$ matrices with positive diagonal entries or [5] and [11] for the subclass of $B$-matrices. For classes of matrices containing $B$-matrices, error bounds for the LCP have also been obtained ([4], [7], [8], [9]). Among these classes of matrices we can mention the $B$-Nekrasov matrices, which will be also considered in this paper.

In some examples, the bound of [5] for $B$-matrices was improved by the bounds of [11]. We present and characterize in Section 2 a subclass of $B$-matrices called strong $B$-matrices, for which the bound of [5] is linear and asymptotically optimal (see Theorem 1) and for which the bound of [11] is worse than or equal to quadratic (see Theorem 3). At the end of Section 2, we also include a family of matrices that are simultaneously strong $B$-matrices and $H$-matrices and for which our bound is 1 and the bound of formula (2.4) of [1] (valid for $H$-matrices with positive diagonal entries) is arbitrarily large. A final example in Section 3 shows that our bound of [5] can improve that of [11] even for $B$-matrices that are not strong $B$-matrices. Finally, Section 4 introduces the class of strong $B$-Nekrasov matrices, which contains strong $B$-matrices and is contained in the class of $B$-Nekrasov matrices. We provide an error bound for the LCP of a strong $B$-Nekrasov matrix that is asymptotically optimal.

\section{A class of $B$-matrices with an asymptotically optimal bound}

The class of $B$-matrices is a subclass of $P$-matrices presented in [13] and has been applied to eigenvalues localization problems ([13], [14]) and to linear complementarity problems ([5], [11]). We recall the definition of a $B$-matrix.

Definition 1 A square real matrix $M=\left(m_{i j}\right)_{1 \leq i, j \leq n}$ is a $B$ - matrix if it has positive row sums and all its off-diagonal entries are bounded above by the corresponding row means, that is, for each $i=1, \ldots, n$,

$$
\sum_{j=1}^{n} m_{i j}>0, \quad \text { and } \quad \frac{1}{n}\left(\sum_{k=1}^{n} m_{i k}\right)>m_{i j} \quad \forall j \neq i .
$$

Given a matrix $M=\left(m_{i j}\right)_{1 \leq i, j \leq n}$, we define for each $i=1, \ldots, n, r_{i}^{+}:=\max \left\{0, m_{i j} \mid j \neq i\right\}$ and we can decompose $M$ into the form $M=B^{+}+C$, where

$$
B^{+}=\left(\begin{array}{ccc}
m_{11}-r_{1}^{+} & \cdots & m_{1 n}-r_{1}^{+} \\
\vdots & & \vdots \\
\vdots & & \vdots \\
m_{n 1}-r_{n}^{+} & \cdots & m_{n n}-r_{n}^{+}
\end{array}\right) \text {and } C=\left(\begin{array}{ccc}
r_{1}^{+} & \cdots & r_{1}^{+} \\
\vdots & & \vdots \\
\vdots & & \vdots \\
r_{n}^{+} & \cdots & r_{n}^{+}
\end{array}\right) \text {. }
$$

Then by Proposition 2.1 of [5], $M$ is a $B$-matrix if and only if $B^{+}=:\left(b_{i j}\right)_{1 \leq i, j \leq n}$ is a strictly diagonally dominant matrix $\left(\left|b_{i i}\right|>\sum_{j \neq i}\left|b_{i j}\right|, i=1, \ldots, n\right)$ with positive diagonal entries. In this paper we introduce the following subclass of $B$-matrices by requiring a stronger diagonal dominant property to $B^{+}$.

Definition 2 Let $M$ be a $B$-matrix and let us consider $M=B^{+}+C$ as in (2). Given $B^{+}=$: $\left(b_{i j}\right)_{1 \leq i, j \leq n}$, we define, for each $i=1, \ldots, n, \beta_{i}:=b_{i i}-\sum_{j \neq i}\left|b_{i j}\right|$. Then we say that $M$ is a strong $B$-matrix if $\beta_{i}>1$ for all $i=1, \ldots, n$. 
Given a complex matrix $M=\left(m_{i j}\right)_{1 \leq i, j \leq n}$, its comparison matrix $\tilde{M}=\left(\tilde{m}_{i j}\right)_{1 \leq i, j \leq n}$ is given by $\tilde{m}_{i j}:=-\left|m_{i j}\right|$ if $i \neq j$ and $\tilde{m}_{i i}:=\left|m_{i i}\right|$ for $i=1, \ldots, n$. Let us recall that $M$ is an $H$-matrix if $\tilde{M}$ is a nonsingular $M$-matrix, that is, if $\tilde{M}^{-1}$ is nonnegative. Error bounds for LCP with $H$-matrices cannot be applied to LCP with strong $B$-matrices because a strong $B$-matrix is not necessarily an $H$-matrix, as the following example shows.

Example 1 Let us consider the matrix

$$
M=\left(\begin{array}{rrrr}
61.1 & 30 & 20 & 10 \\
-20 & 37.5 & 0 & -16 \\
0 & -40 & 51.5 & -10 \\
50 & 50 & 10 & 91.5
\end{array}\right)
$$

The decomposition $M=B^{+}+C$ with $B^{+}$and $C$ given in (2) leads to

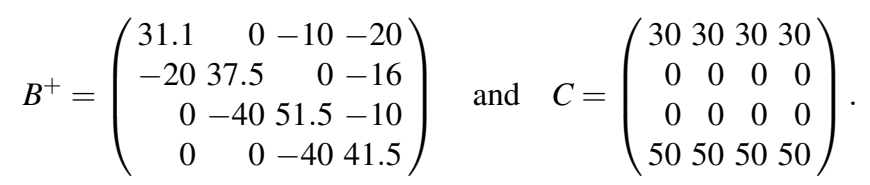

Observe that $M$ is a $B$-matrix and even a strong $B$-matrix. However, $M$ is not an $H$-matrix because its comparison matrix $\tilde{M}$ has an inverse with nonpositive entries.

The following result characterizes strong $B$-matrices.

Proposition $1 M:=\left(m_{i j}\right)_{1 \leq i, j \leq n}$ is a strong $B$-matrix if and only if for each $i=1, \ldots, n$,

$$
\sum_{j=1}^{n} m_{i j}>1 \quad \text { and } \quad \frac{1}{n}\left(\sum_{j=1}^{n} m_{i j}-1\right)>m_{i k} \quad \forall k \neq i .
$$

Proof Let us assume first that $M$ is a strong $B$-matrix. Then $\beta_{i}=b_{i i}-\sum_{j \neq i}\left|b_{i j}\right|>1$, for all $i=1, \ldots, n$, and $B^{+}=\left(b_{i j}\right)_{1 \leq i, j \leq n}$ is given in (2). Taking into account that $r_{i}=\max \left\{0, m_{i j} \mid j \neq\right.$ $i\}$, we have that $r_{i}^{+} \geq m_{i j}$ for all $j \neq i$, and we derive from the previous formula

$$
1<m_{i i}-r_{i}^{+}-\sum_{j \neq i}\left(r_{i}^{+}-m_{i j}\right)=\sum_{j=1}^{n} m_{i j}-n r_{i}^{+}
$$

and so we conclude that $\sum_{j=1}^{n} m_{i j}>1+n r_{i}^{+} \geq 1$. From (4) we also have $\sum_{j=1}^{n} m_{i j}-1>$ $n r_{i}^{+} \geq n m_{i k}$ for all $k \neq i$ and (3) holds.

Let us now assume that (3) holds. Clearly, (3) implies that $M$ is a $B$-matrix. Since $r_{i}^{+} \geq$ $m_{i j}$ for all $j \neq i$,

$$
\beta_{i}=m_{i i}-r_{i}^{+}-\sum_{j \neq i}\left(r_{i}^{+}-m_{i j}\right)=\sum_{j=1}^{n} m_{i j}-n r_{i}^{+} .
$$

If $r_{i}^{+}=0$, then by (5) and (3), $\beta_{i}=\sum_{j=1}^{n} m_{i j}>1$. Finally, if $r_{i}^{+} \neq 0$, then there exists $k \neq i$ such that $r_{i}^{+}=m_{i k}>0$. Then, by (5) and (3) we obtain $\beta_{i}=\sum_{j=1}^{n} m_{i j}-n m_{i k}=$ $n\left(\frac{1}{n} \sum_{j=1}^{n} m_{i j}-m_{i k}\right)>n\left(\frac{1}{n}\right)=1$ and $M$ is a strong $B$-matrix. 
Example 2 Now we present some examples arising in practical applications. Given the tridiagonal $n \times n$ matrix

$$
M=\left(\begin{array}{ccccc}
b+\alpha \sin \left(\frac{1}{n}\right) & c & & & \\
& a b+\alpha \sin \left(\frac{2}{n}\right) & c & & \\
& \ddots & \ddots & \ddots & \\
& & \ddots & \ddots & c \\
& & & a & b+\alpha \sin (1)
\end{array}\right)
$$

the $\operatorname{LCP}(M, q)$ with various $q$ in an interval vector arises from the finite difference method for free boundary problems (cf. [15], [1]). Observe that, for $b>3, a=c=-1, \alpha=0$, the corresponding family is formed by strong $B$-matrices and also for $b=3, a=c=-1, \alpha>0$. In fact, the decomposition (2) for these matrices is of the form $M=B^{+}+0$ and $B^{+}$satisfies the properties of Definition 2. Besides, for any $k_{1}, \ldots, k_{n} \geq 0$, we can form matrices

$$
C=\left(\begin{array}{ccc}
k_{1} & \cdots & k_{1} \\
\vdots & & \vdots \\
\vdots & & \vdots \\
k_{n} & \cdots & k_{n}
\end{array}\right)
$$

and $M+C$ is again a strong $B$-matrix by Definition 2 .

In this section we shall prove that the bound of Theorem 2.2 of [5] is asymptotically optimal for the class of strong $B$-matrices. For this purpose, we consider the following family of $n \times n$ strong $B$-matrices $(n \geq 2)$,

$$
M_{m}=\left(\begin{array}{cccc}
m+k & m & \cdots & m \\
1 & 1+k & \cdots & 1 \\
\vdots & \vdots & \vdots & \vdots \\
\vdots & \vdots & \vdots & \vdots \\
1 & 1 & \cdots & 1+k
\end{array}\right)
$$

where $m$ is a positive integer and $k>1$. Observe that, if $k \in(0,1]$, then $M_{m}$ is a $B$-matrix but it is not a strong $B$-matrix.

First, we recall some notations for the linear complementarity problem (1). Its solution $x^{*}$ is unique if and only if $M$ is a $P$-matrix. In this case, by Theorem 2.3 of [1],

$$
\left\|x-x^{*}\right\|_{\infty} \leq \max _{d \in[0,1]^{n}}\left\|(I-D+D M)^{-1}\right\|_{\infty}\|r(x)\|_{\infty},
$$

where $I$ is the identity matrix, $D$ is the diagonal matrix $\operatorname{diag}\left(d_{i}\right)$ with $0 \leq d_{i} \leq 1$ for all $i=1, \ldots, n$, and $r(x):=\min (x, M x+q)$, where the min operator denotes the componentwise minimum of two vectors. If $M$ is a $B$-matrix and $\beta_{i}, i=1, \ldots, n$, are defined as in Definition 2 , let us denote by

$$
\beta:=\min _{i \in\{1, \ldots, n\}}\left\{\beta_{i}\right\}
$$

Then, by Theorem 2.2 of [5],

$$
\max _{d \in[0,1]^{n}}\left\|(I-D+D M)^{-1}\right\|_{\infty} \leq \frac{n-1}{\min \{\beta, 1\}} .
$$


Theorem 1 For strong B-matrices, the bound (9) is asymptotically optimal and it is equal to $n-1$.

Proof First observe that for strong $B$-matrices the bound (9) is equivalent to

$$
\max _{d \in[0,1]^{n}}\left\|(I-D+D M)^{-1}\right\|_{\infty} \leq n-1,
$$

because of (8) and $\beta>1$. Let us consider the family of matrices $M_{m}$ given by (7), with $m$ a positive integer and $k>1$, and the particular choice in the left side of (9) given by $d=(1, \ldots, 1)^{T}$, which corresponds to the diagonal matrix $D=I$. So, with this choice, we have the following inequality

$$
\max _{d \in[0,1]^{n}}\left\|\left(I-D+D M_{m}\right)^{-1}\right\|_{\infty} \geq\left\|M_{m}^{-1}\right\|_{\infty} .
$$

Observe that $M_{m}$ can be written in the form (2) as $M_{m}=K+u e^{T}$ where

$$
K:=\left(\begin{array}{cccc}
k & 0 & \cdots & 0 \\
0 & k & \cdots & 0 \\
\vdots & \vdots & \vdots & \vdots \\
\vdots & \vdots & \vdots & \vdots \\
0 & 0 & \cdots & k
\end{array}\right), \quad u:=\left(\begin{array}{c}
m \\
1 \\
\vdots \\
\vdots \\
1
\end{array}\right), \quad e:=\left(\begin{array}{c}
1 \\
1 \\
\vdots \\
\vdots \\
1
\end{array}\right) \text {. }
$$

Then we have

$$
M_{m}=K\left(I+K^{-1} u e^{T}\right)=K\left(I+u_{k} e^{T}\right),
$$

where $u_{k}:=(m / k, 1 / k, \ldots, 1 / k)^{T}$. Then, since $e^{T} u_{k} \neq-1$, by the Sherman-Morrison formula (see formula (2.1.5) of page 65 of [10]), we obtain from (12)

$$
M_{m}^{-1}=\left(I+u_{k} e^{T}\right)^{-1} K^{-1}=\left(I-\frac{u_{k} e^{T}}{1+e^{T} u_{k}}\right) K^{-1}
$$

and so

$$
M_{m}^{-1}=\left(\begin{array}{cccc}
1-\frac{m}{m+n+k-1} & \frac{-m}{m+n+k-1} & \cdots & \frac{-m}{m+n+k-1} \\
\frac{m-1}{m+n+k-1} & 1-\frac{1}{m+n+k-1} & \cdots & \frac{-1}{m+n+k-1} \\
\vdots & \vdots & \vdots & \vdots \\
\vdots & \vdots & \vdots & \vdots \\
\frac{-1}{m+n+k-1} & \frac{-1}{m+n+k-1} & \cdots & 1-\frac{1}{m+n+k-1}
\end{array}\right) .
$$

Then $\left\|M_{m}^{-1}\right\|_{\infty}=1-\frac{m}{m+n+k-1}+\frac{(n-1) m}{m+n+k-1}=\frac{n+(k-1)+(n-1) m}{m+n+k-1}$ and we derive

$$
\lim _{m \rightarrow \infty}\left\|M_{m}^{-1}\right\|=n-1 \text {. }
$$

By (11) and (13), $\lim _{m \rightarrow \infty} \max _{d \in[0,1]^{n}}\left\|\left(I-D+D M_{m}\right)^{-1}\right\|_{\infty}=n-1$ and (10) is asymptotically optimal.

Let us illustrate the asymptotic optimality of the previous result with some particular values of $n, k, m$ for the $n \times n$ matrix $M_{m}$ of (7). A lower bound for $\max _{d \in[0,1]^{n}} \|(I-D+$ $\left.D M_{m}\right)^{-1} \|_{\infty}$ is $\left\|M_{m}^{-1}\right\|_{\infty}$ by (11) and in the line previous to (13) we have seen that $\left\|M_{m}^{-1}\right\|_{\infty}=$ $1-\frac{m}{m+n+k-1}+\frac{(n-1) m}{m+n+k-1}=\frac{n+(k-1)+(n-1) m}{m+n+k-1}$. For $n=10$ and $k=2$, we obtain that this lower bound is $\frac{20}{12}$ for $m=1, \frac{101}{21}$ for $m=10, \frac{911}{111}$ for $m=100$ and $\frac{9011}{1011}$ for $m=1000$, which shows the approximation to our upper bound $n-1=9$ of (10).

We now present a family of $2 \times 2$ matrices of Example 2 that are simultaneously strong $B$-matrices and $H$-matrices. For these matrices our bound (10) is 1 and the bound of formula (2.4) of [1] (valid for $H$-matrices with positive diagonal entries) is arbitrarily large. 
Example 3 Let us consider $2 \times 2$ matrices of Example 2 with $b=4, a=c=-1, \alpha=0$ and $k_{1}=k_{2}=k>1$. So our matrices are of the form

$$
M=\left(\begin{array}{ll}
4+k & k-1 \\
k-1 & 4+k
\end{array}\right) .
$$

These strong $B$-matrices are also $H$-matrices because the comparison matrix

$$
\tilde{M}=\left(\begin{array}{cc}
4+k & -k+1 \\
-k+1 & 4+k
\end{array}\right)
$$

has nonnegative inverse

$$
\tilde{M}^{-1}=\left(\begin{array}{cc}
\frac{4+k}{5(3+2 k)} & \frac{k-1}{5(3+2 k)} \\
\frac{k-1}{5(3+2 k)} & \frac{4+k}{5(3+2 k)}
\end{array}\right) .
$$

Our bound (10) is $n-1=1$. Let us now consider the bound of (2.4) of [1]:

$$
\max _{d \in[0,1]^{n}}\left\|(I-D+D M)^{-1}\right\|_{\infty} \leq\left\|\tilde{M}^{-1} \max (\Lambda, I)\right\|_{\infty},
$$

where $\Lambda$ is the diagonal part of $M\left(\Lambda:=\operatorname{diag}\left(m_{i i}\right)\right)$ and $\max (\Lambda, I):=\operatorname{diag}\left(\max \left\{m_{11}, 1\right\}, \ldots, \max \left\{m_{n n}, 1\right\}\right)$. For our matrices $M, \max _{d \in[0,1]^{n}}\left\|(I-D+D M)^{-1}\right\|_{\infty}=\frac{4}{5}+\frac{k}{5}$, which is greater than 1 and can be arbitrarily large.

\section{Comparisons with another recent bound}

In [11] the authors provided for a $B$-matrix $M$ an upper bound for $\max _{d \in[0,1]^{n}} \|(I-D+$ $D M)^{-1} \|_{\infty}$ different from (9) and they showed some examples where this bound (presented in Theorem 4 of [11]) improves (9). We are now going to prove that for strong $B$-matrices the bound (9) (or its equivalent form (10) as shown in Theorem 1) is better than the bound of Theorem 4 of [11]. We shall prove in Theorem 2 that the bound of Theorem 4 of [11] is worse than or equal to quadratic $n(n-1)$, in contrast with our linear bound $n-1$ of (10). Previously we recall Theorem 4 of [11].

Theorem 2 Let $M=\left(m_{i j}\right)_{1 \leq i, j \leq n}$ be a $B$ - matrix with the form $M=B^{+}+C$, where $B^{+}:=$ $\left(b_{i j}\right)_{1 \leq i, j \leq n}$ and $C$ are given in (2). Then

$\max _{d \in[0,1]^{n}}\left\|(I-D+D M)^{-1}\right\|_{\infty} \leq \frac{n-1}{\min \left\{\bar{\beta}_{1}, 1\right\}}+\sum_{i=2}^{n} \frac{n-1}{\min \left\{\bar{\beta}_{i}, 1\right\}} \prod_{j=1}^{i-1}\left(1+\frac{1}{\bar{\beta}_{j}} \sum_{k=j+1}^{n}\left|b_{j k}\right|\right)=: b_{n}$,

where for each $i=1, \ldots, n$,

$$
\bar{\beta}_{i}:=b_{i i}-l_{i}\left(B^{+}\right) \sum_{j=i+1}^{n}\left|b_{i j}\right| \quad \text { and } \quad l_{i}\left(B^{+}\right):=\max _{i \leq k \leq n}\left\{\frac{1}{\left|b_{k k}\right|} \sum_{j=i, j \neq k}^{n}\left|b_{k j}\right|\right\} .
$$

Let us now provide a lower bound for the bound of Theorem 2 in the case of strong $B$-matrices.

Theorem 3 Let $M=\left(m_{i j}\right)_{1 \leq i, j \leq n}$ be a strong B-matrix. Then the bound $b_{n}$ of $(14)$ satisfies

$$
b_{n} \geq n(n-1)
$$


Proof With the notations of Theorem 2, let us observe that for each $i=1, \ldots, n, l_{i}\left(B^{+}\right)<1$ (see (11) of [11]). Therefore $\bar{\beta}_{i} \geq \beta_{i}$, for each $i=1, \ldots, n$. Since $M$ is a strong $B$-matrix, for $i=1, \ldots, n, \beta_{i}>1$, which implies that $\bar{\beta}_{i}>1$ and $\operatorname{so}, \min \left\{\bar{\beta}_{i}, 1\right\}=1$. Then, since $\prod_{j=1}^{i-1}\left(1+\frac{1}{\bar{\beta}_{j}} \sum_{k=j+1}^{n}\left|b_{j k}\right|\right) \geq 1$, we can deduce that $b_{n} \geq n-1+\sum_{i=2}^{n}(n-1) .1=n(n-1)$.

Although theorems 1 and 3 only hold for strong $B$-matrices, the bound (9) can be sharp and even better than (14), also for $B$-matrices that are not strong $B$-matrices. In the following example we consider a $B$-matrix that is not strong and we shall compare the bound (9) with (14). Let us consider the matrix

$$
M=\left(\begin{array}{rrrr}
60.5 & 30 & 20 & 10 \\
-20 & 40 & 0 & -16 \\
0 & -40 & 51 & -10 \\
50 & 50 & 10 & 91
\end{array}\right)
$$

The decomposition $M=B^{+}+C$ with $B^{+}$and $C$ given in (2) leads to

$$
B^{+}=\left(\begin{array}{rrrr}
30.5 & 0 & -10 & -20 \\
-20 & 40 & 0 & -16 \\
0 & -40 & 51 & -10 \\
0 & 0 & -40 & 41
\end{array}\right) \quad \text { and } \quad C=\left(\begin{array}{rrrr}
30 & 30 & 30 & 30 \\
0 & 0 & 0 & 0 \\
0 & 0 & 0 & 0 \\
50 & 50 & 50 & 50
\end{array}\right)
$$

Observe that $M$ is a $B$-matrix (because $B^{+}$is strictly diagonally dominant with positive diagonal entries) and that it is not a strong $B$-matrix (because $\beta=\beta_{1}=0.5$ ). One can check that the bound of Theorem 4 of [11] (that is, the bound $b_{n}$ of (14)) is in this case

$$
\max _{d \in[0,1]^{n}}\left\|(I-D+D M)^{-1}\right\|_{\infty} \leq b_{n}=445.321 .
$$

Let us now compute our bound (9). Taking into account that $n=4$ and $\beta_{1}=0.5, \beta_{2}=4, \beta_{3}=$ $\beta_{4}=1$, (and so, $\beta=0.5$ by ( 8 ), we deduce that (9) is now $3 / 0.5=6$.

\section{Strong $B$-Nekrasov matrices and asymptotically optimal bounds}

Let us recall the definition of a Nekrasov matrix. For this purpose, we need some notations. If $M=\left(m_{i j}\right)_{1 \leq i, j \leq n}$ is a complex matrix with $m_{i i} \neq 0$ for all $i=1, \ldots, n$, let us define

$$
h_{1}(M):=\sum_{j \neq 1}\left|m_{1 j}\right|, h_{i}(M):=\sum_{j=1}^{i-1}\left|m_{i j}\right| \frac{h_{j}(M)}{\left|m_{j j}\right|}+\sum_{j=i+1}^{n}\left|m_{i j}\right|, \quad i=2, \ldots, n .
$$

Then $M$ is a Nekrasov matrix if $\left|m_{i i}\right|>h_{i}(M)$ for all $i=1, \ldots, n$ (cf. [16]). It is well-known that Nekrasov matrices are nonsingular matrices.

The following definition recalls the concept of $B$-Nekrasov matrix, which was introduced in [8], and introduces the new definition of strong $B$-Nekrasov matrices.

Definition 3 A real matrix $M$ is a $B$-Nekrasov matrix if $M=B^{+}+C$, where $B^{+}$and $C$ are given in (2) and $B^{+}$is a Nekrasov $Z$-matrix with all diagonal entries positive. If $B^{+}-I$ is a Nekrasov $Z$-matrix with all diagonal entries positive, then we say that $M$ is a strong $B$-Nekrasov matrix. 
By Remark 1 of [8], $B$-matrices are $B$-Nekrasov matrices and, by Proposition 1 of [8], $B$ Nekrasov matrices are $P$-matrices. Since a strong $B$-matrix $M$ can be written $M=B^{+}+C$ as in (2) and satisfies that $B^{+}-I$ is a strictly diagonally dominant matrix with positive diagonal entries, we can deduce that a strong $B$-matrix is a strong $B$-Nekrasov matrix.

The following result shows that a strong $B$-Nekrasov matrix is also a $B$-Nekrasov matrix.

Proposition 2 If $M$ is a strong B-Nekrasov matrix, then M is also B-Nekrasov.

Proof It is sufficient to prove that if $B^{+}-I$ is a Nekrasov $Z$-matrix with positive diagonal entries, then $B^{+}=\left(b_{i j}\right)_{1 \leq i, j \leq n}$ is a Nekrasov $Z$-matrix with positive diagonal entries. So, we assume that

$$
b_{i i}-1>h_{i}\left(B^{+}-I\right), \quad i=1, \ldots, n,
$$

and let us prove that

$$
b_{i i}>h_{i}\left(B^{+}\right), \quad i=1, \ldots, n .
$$

Since $b_{i i}>b_{i i}-1$ for all $i=1, \ldots, n$, in order to prove (18) from (17), we shall prove that

$$
h_{i}\left(B^{+}-I\right) \geq h_{i}\left(B^{+}\right), \quad i=1, \ldots, n .
$$

Let us prove (19) by induction on $i$. Since $h_{1}\left(B^{+}-I\right)=\sum_{j \neq 1}\left|b_{1 j}\right|=h_{1}\left(B^{+}\right)$, (19) holds for $i=1$. Let us now assume that (19) holds for all $j<i$ and let us prove it for $i$. Then

$$
h_{i}\left(B^{+}-I\right)=\sum_{j=1}^{i-1}\left|b_{i j}\right| \frac{h_{j}\left(B^{+}-I\right)}{b_{j j}-1}+\sum_{j=i+1}^{n}\left|b_{i j}\right| .
$$

Taking into account the induction hypothesis and that $b_{j j}-1<b_{j j}$, we obtain

$$
h_{i}\left(B^{+}-I\right) \geq \sum_{j=1}^{i-1}\left|b_{i j}\right| \frac{h_{j}\left(B^{+}\right)}{b_{j j}}+\sum_{j=i+1}^{n}\left|b_{i j}\right|=h_{i}\left(B^{+}\right)
$$

and the induction holds.

The converse of the previous proposition does not hold as the following example shows. Any matrix

$$
M_{k}=\left(\begin{array}{cc}
k & -k+1 \\
-k+1 & k
\end{array}\right), \quad k \geq 2,
$$

is $B$-Nekrasov because $M_{k}=B_{k}^{+}+C_{k}$ with $B_{k}^{+}=M_{k}$ and $C_{k}=0$ and it can be checked that $B_{k}=M_{k}$ is Nekrasov. However, $M_{k}$ is not strong $B$-Nekrasov because

$$
B_{k}^{+}-I=\left(\begin{array}{cc}
k-1 & -k+1 \\
-k+1 & k-1
\end{array}\right)
$$

is singular and so, $B_{k}^{+}-I$ can not be Nekrasov.

It is well-known that a complex matrix $A$ is an $H$-matrix if there exists a diagonal matrix $W=\operatorname{diag}\left(w_{i}\right)$ such that $A W$ is strictly diagonal dominant. Observe that the diagonal matrix $W$ can be taken with positive diagonal entries (it is sufficient using $\operatorname{diag}\left(\left|w_{i}\right|\right)$ ). It is known that a Nekrasov matrix is an $H$-matrix (see [16] and p. 5021 of [3]). So, given a $B$-Nekrasov matrix $M$, there exists a diagonal matrix $W$ with positive diagonal entries such that $B^{+} W$ is a strictly diagonally dominant $Z$-matrix (where $B^{+}$is given by (2)). Then this holds, in particular, for strong $B$-Nekrasov matrices. Since a strong $B$-matrix satisfies that $B^{+}-I$ is 
a strictly diagonally dominant matrix with positive diagonal entries, we can deduce that a strong $B$-matrix is a strong $B$-Nekrasov matrix.

In Theorem 2 of [8], we obtained an error bound for the LCP of a $B$-Nekrasov matrix $A=B^{+}+C$ satisfying certain hypotheses that allowed us to construct a particular diagonal matrix $W$ such that $B^{+} W$ is a strictly diagonally dominant $Z$-matrix. As we have recalled in the previous paragraph, for any $B$-Nekrasov matrix $A\left(=B^{+}+C\right)$, there exists a diagonal matrix $W$ with positive diagonal entries such that $B^{+} W$ is a strictly diagonally dominant $Z$-matrix. The same proof of Theorem 2 of [8] can be used to prove the following result, which does not require any additional hypothesis on the $B$-Nekrasov matrices.

Theorem 4 Let $M=\left(m_{i j}\right)_{1 \leq i, j \leq n}$ be a B-Nekrasov matrix, let $B^{+}$be the matrix of $(2)$ and let $W=\operatorname{diag}\left(w_{i}\right)$ be the diagonal matrix with positive diagonal entries such that $\bar{B}:=B^{+} W=$ $\left(\bar{b}_{i j}\right)_{1 \leq i, j \leq n}$ is a strictly diagonally dominant $Z$-matrix. Let $\beta_{i}:=\bar{b}_{i i}-\sum_{j \neq i}\left|\bar{b}_{i j}\right|$ and $\delta_{i}:=\frac{\beta_{i}}{w_{i}}$, for $i=1, \ldots, n$ and $\delta=\min _{i}\left\{\delta_{i}\right\}$. Then

$$
\max _{d \in[0,1]^{n}}\left\|(I-D+D M)^{-1}\right\|_{\infty} \leq \frac{(n-1) \max _{i}\left\{w_{i}\right\}}{\min \{1, \delta\} \min _{i}\left\{w_{i}\right\}}
$$

Now, we shall provide an error bound for the LCP of a strong $B$-Nekrasov matrix $M$.

Theorem 5 Let $M=\left(m_{i j}\right)_{1 \leq i, j \leq n}$ be a strong B-Nekrasov matrix, let $B^{+}$be the matrix of (2) and let $W=\operatorname{diag}\left(w_{i}\right)$ be the diagonal matrix with positive diagonal entries such that $\left(B^{+}-I\right) W$ is a strictly diagonally dominant Z-matrix. Then

$$
\max _{d \in[0,1]^{n}}\left\|(I-D+D M)^{-1}\right\|_{\infty} \leq \frac{(n-1) \max _{i}\left\{w_{i}\right\}}{\min _{i}\left\{w_{i}\right\}}
$$

Moreover, the bound (21) is asymptotically optimal.

Proof Since $M$ is a strong $B$-matrix, $B^{+}-I$ is a Nekrasov matrix and so it is an $H$-matrix. Therefore there exists a diagonal matrix $W$ with positive diagonal entries such that $\left(B^{+}-\right.$ $I) W$ is a strictly diagonally dominant $Z$-matrix. Then $B^{+} W$ is also a strictly diagonally dominant $Z$-matrix and, since a strong $B$-Nekrasov matrix is a $B$-Nekrasov matrix, we can apply Theorem 4 with $\bar{B}:=B^{+} W$ and (20) holds. Since $\left(B^{+}-I\right) W=\bar{B}-W$ is also a strictly diagonally dominant $Z$-matrix, $\beta_{i}-w_{i}>0$ for all $i=1, \ldots, n$. This implies that $\delta_{i}:=\frac{\beta_{i}}{w_{i}}>1$ for all $i=1, \ldots, n$. If $\delta=\min _{i}\left\{\delta_{i}\right\}$, then $\min \{1, \delta\}=1$ and so (20) becomes (21).

Now, let us consider the family of strong $B$-Nekrasov matrices $M_{m}$ given by (7) with $m$ a positive integer and $k>1$, and the particular choice in the left side of (21) given by $d=(1, \ldots, 1)^{T}$, which corresponds to the diagonal matrix $D=I$. So, with this choice we have (11). Observe that if we write $M=B^{+}+C$ as in (2), then $B^{+}$is the diagonal matrix whose diagonal entries are equal to $k$. So, $B^{+}-I$ is a strictly diagonally dominant $Z$-matrix and we can choose the matrix $W=I$ to obtain the formula (21). In this case the right side of (21) is $n-1$. Finally, since (11) and (13) hold, we see that (21) is asymptotically optimal.

Acknowledgements The authors wish to thank the anonymous referees for their valuable suggestions to improve the paper. This work was partially supported by the Spanish Research Grant MTM2015-65433-P (MINECO/FEDER), Gobierno de Aragón and Fondo Social Europeo. 


\section{References}

1. X. Chen and S. Xiang, Computation of error bounds for P-matrix linear complementarity problems. Math. Program., Ser. A, 106 (2006), 513-525.

2. R. W. Cottle, J.-S. Pang and R. E. Stone, The Linear Complementarity Problems. Academic Press, Boston MA, 1992.

3. L. Cvetković, P-F. Dai, K. Doroslovaški, Y-T. Li, Infinity norm bounds for the inverse of Nekrasov matrices. Appl. Math. Comput. 219 (2013), 5020-5024.

4. P.-F. Dai, Error bounds for linear complementarity problems of DB-matrices. Linear Algebra Appl. 434 (2011), 830-840.

5. M. García-Esnaola and J. M. Peña, Error bounds for linear complementarity problems for B-matrices Appl. Math. Lett. 22 (2009), 1071-1075.

6. M. García-Esnaola and J. M. Peña, A comparison of error bounds for linear complementarity problems of H-matrices. Linear Algebra Appl. 433 (2010), 956-964.

7. M. García-Esnaola and J. M. Peña, Error bounds for linear complementarity problems of $B^{S}$-matrices. Appl. Math. Lett. 25 (2012), 1379-1383.

8. M. García-Esnaola and J. M. Peña, $B$-Nekrasov matrices and error bounds for linear complementarity problems. Numer. Algor. 72 (2016), 435-445.

9. M. García-Esnaola and J. M. Peña, $B_{\pi}^{R}$-matrices and error bounds for linear complementarity problems. Calcolo 54 (2017), 813-822.

10. G. H. Golub and C. F. Van Loan, Matrix Computations (Fourth edition). The Johns Hopkins University Press, Baltimore, 2013

11. C. Li and Y. Li, Note on error bounds for linear complementarity problems for $B$-matrices. Appl. Math. Lett. 57 (2016), 108-113.

12. R. Mathias and J.-S. Pang, Error bounds for the linear complementarity problem with a $P$-matrix. Linear Algebra Appl. 132 (1990), 123-136.

13. J. M. Peña, A class of $P$-matrices with applications to the localization of the eigenvalues of a real matrix. SIAM J. Matrix Anal. Appl. 22 (2001), 1027-1037.

14. J. M. Peña, On an alternative to Gerschgorin circles and ovals of Cassini. Numer. Math. 95 (2003), 337-345.

15. U. Schäfer, An enclosure method for free boundary problems based on a linear complementarity problem with interval data. Numer. Funct. Anal. Optim. 22 (2001), 991-1011.

16. T. Szulc, Some remarks on a theorem of Gudkov, Linear Algebra Appl. 225 (1995), 221-235. 